\title{
Kombination verbessert den Therapieerfolg
}

Fragstellung: Das Ziel dieser Studie war es herauszufinden, ob die kognitive Verhaltenstherapie (KVT) in Kombination mit einer Expositionstherapie einen Zusatznutzen bei der Behandlung der verlängerten Trauerreaktion hat.

Hintergrund: Etwa $10 \%$ der Menschen, die den Verlust eines geliebten Menschen erlebt haben, entwickeln eine verlängerte Trauerreaktion. Daher gibt es den Vorschlag, in der Überarbeitung der Internationalen statistischen Klassifikation der Krankheiten (ICD 11) eine neue Diagnose einzuführen: „ die verlängerte Trauererkrankung“. Sie beinhaltet unter anderem die anhaltende Sehnsucht nach dem Verstorbenen, den damit verbundenen emotionalen Schmerz und somit Schwierigkeiten bei der Annahme des Todes oder Schwierigkeiten, neue Lebensziele oder Aktivitäten zu entwickeln. In verschiedenen Studien zeigte sich, dass zielgerichtete KVT (bestehend u.a. aus Wiederaufgreifen des Todes, Förderung positiver Erinnerungen und Erarbeiten von neuen Zielen) als Therapieform wirksam ist. In anderen Studien wurden Expositionen (z. B. Erinnerung an den Tod des Verstorben und Emotionen an den Verstorbenen) als Teil der Therapie eingesetzt. Diese Studien belegen, dass die expositionsbasierte Therapie ein effektives Mittel zur Linderung der Symptome der verlängerten Trauerreaktion sein könnte. Jedoch kann der expositionsbasierte Ansatz ausgeprägte Verzweiflung während des Wiedererlebens hervorrufen. Obwohl diese Verzweiflung nicht dauerhaft anhält, ist es wichtig, zu verstehen, wie wesentlich die Expositionstherapie für eine

Bryant RA, Kenny L, Joscelyne A et al. Treating prolonged grief disorder: a randomized clinical trial. JAMA Psychiatry 2014; 71: $1332-9$ effektiv behandelte verlängerte Trauerreaktion ist.

Patienten und Methodik: Die Studie schloss 80 Patien- ten mit verlängerter Trauerreaktion ein, bei denen der Trauerfall mindestens zwölf Monate zurücklag. Alle Patienten erhielten eine Therapie aus zehn wöchentlichen zweistündigen Gruppentherapiesitzungen und vier wöchentlichen einstündigen Einzeltherapiesitzungen. In der Gruppe KVT plus Expositionstherapie wurden in den Einzeltherapiesitzungen Erinnerungen an den Verstorben thematisiert, in der anderen Gruppe fanden in den Einzeltherapiesitzungen supportive Gespräche mit vom Patienten selbst gewählten Themen statt. Die Inhalte der Gruppensitzungen waren in beiden Gruppen identisch. Zur Bewertung der Ausgangssymptomatik und der Veränderung unter der Therapie wurden verschiedene Fragebögen verwendet und ein klinisches, halbstrukturiertes Interview zur Beurteilung der komplizierten Trauerreaktion durchgeführt.

Ergebnisse: In der Gruppe, die KVT plus Expositionstherapie erhielt, zeigte sich eine signifikante Verbesserung der Trauersymptomatik, der depressiven Symptomatik, der negativen Sichtweise auf die Welt und der negativen Selbstwahrnehmung im Vergleich mit der Gruppe, die nur KVT erhielt. Zudem war in der Gruppe mit Expositionstherapie die psychische und soziale Funktion signifikant besser. In Bezug auf den Follow-upDiagnosestatus wurde die Number-needed-to-treat mit 4,32 angegeben.

Schlussfolgerungen: Viele Studien zeigen, dass Vermeidungsstrategien, welche die Patienten mit einer verlängerten Trauerreaktion oft anwenden, ein Hauptgrund für die nicht beherrschbaren Symptome der verlängerten Trauer sein könnten. In dieser Studie zeigte sich, dass kognitive Verhaltenstherapie mit Exposition zu einer optimaleren Steuerung, besseren Kontrolle und Reduktion der Symptome der verlängerten Trauererkrankung führt.

\section{- Kommentar von Kristin Seiler, Erlangen}

\section{Expositionstherapie stellt ein wesentliches Therapieelement dar}

Die Studie erfüllt mit einem JADAD-Score von vier Punkten gute methodische Ansprüche. Sie zeigt, dass sich die Symptome einer verlängerten Trauerreaktion durch Exposition mit dem Tod des Verstorben und Erinnerungen an ihn zusätzlich zur KVT besser beherrschen lassen. Die Bedeutung dieser Erkrankung könnte in den nächsten Jahren zunehmen und sicha uch im Behandlungsalltag widerspiegeln. So können sich somatische Komplikationen wie Herz-Kreislauf- oder Krebserkrankungen, aber auch psychische Störungen wie Suizidalität entwickeln [1]. Daher ist es wichtig, ein wirkungsvolles Konzept für die Diagnostik und psychotherapeutische Behandlung zu erarbeiten, um eine effektive Therapie anzubieten und so die Lebensqualität und Funktionalität der Betroffenen langfristig zu steigern. Weitere Studien zur Therapieoptimierung und zur Erstellung detaillierter Therapiekonzepte sind notwendig.

\author{
Referenz: \\ 1. Prigerson HG et al. Am J Psychiatry 1997; 154: 616-23
}

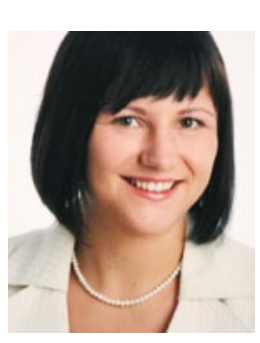

Dr. med. Kristin Seiler, Erlangen

Universitätsklinikum Erlangen, Psychiatrie

E-Mail:kristin.seiler@uk-erlangen.de 